\title{
Fructan Contents in Australian Wheat Varieties Released Over the Last 150 Years
}

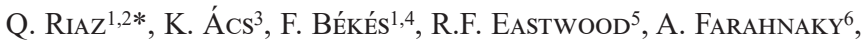 \\ M. MAJZOOBI ${ }^{6}$ and C.L. BlanCHARD ${ }^{1,2}$ \\ ${ }^{1}$ School of Biomedical Sciences, Charles Sturt University, Wagga Wagga, NSW 2650, Australia \\ ${ }^{2}$ Australian Research Council (ARC) Industrial Transformation Training Centre (ITTC) \\ for Functional Grains, Graham Centre for Agricultural Innovation, Charles Sturt University, \\ Wagga Wagga, New South Wales, 2650, Australia \\ ${ }^{3}$ Cereal Research Non-profit Ltd., P.O. Box 391, H-6701 Szeged, Hungary \\ ${ }^{4}$ FBFD PTY LTD, Sydney, Australia \\ ${ }^{5}$ Australian Grain Technologies, Wagga Wagga, New South Wales, Australia \\ ${ }^{6}$ School of Science, RMIT University, Bundoora West Campus, \\ Plenty Road, Melbourne, VIC, 3083, Australia
}

(Received 30 June 2019;

Accepted 8 August 2019)

\begin{abstract}
Grain fructans play an important role in the physiology of wheat plants and also impact on the health of consumers of wheat-based products. Given the potential economic importance of fructan levels, if genetic variability could be identified for this trait, it may be a potentially useful breeding target for developing climate-resilient and nutritionally enhanced wheat varieties. The aim of the current study was to screen 78 genetically diverse Australian wheat varieties released between 1860 and 2015 to determine if historic breeding targets have resulted in changes in fructan levels and to identify potential breeding parents for the development of varieties with specific fructan levels. The impact of seasonal conditions on grain fructan levels were also investigated. Analysis of the varieties in this study indicated that historic breeding targets have not impacted on grain fructan levels. Fructan content in flours varied between 1.01 to $2.27 \%$, showing some variation among the varieties. However, a significant variation in fructan levels was observed between different harvest years (mean values for 2015 and 2016 samples were 1.38 and 1.74\%, respectively). While large variations in fructan contents of different varieties were not found, there were some varieties with consistently higher or lower fructan contents which could be used to breed varieties with specific fructan levels.
\end{abstract}

Keywords: fructan, FODMAP, irritable bowel syndrome, wheat flour, health benefits

\section{Introduction}

Wheat is the third most important cereal after maize and rice based on global production (Shewry et al. 2013). Wheat carbohydrates are considered as a major source of energy in the everyday diet. Wheat is also rich in other nutrient components including proteins, fibre, lipids, minerals, vitamins and bioactive compounds, all of which contribute to a

*Corresponding author; E-mails: qriaz@csu.edu.au, qurat.rp@gmail.com 
healthy diet (Shewry and Hey 2015; Belderok et al. 2000). Due to the widespread consumption and importance of wheat as a dietary source of energy, there has been an increasing interest in exploring the health benefits of wheat and wheat-based foods.

In cereals, fructans are categorized as water soluble storage carbohydrates. Being a source of carbon reserve in plants, fructans have a major functional role in plant physiology (Van den Ende and El-Esawe 2014). In wheat, fructans play a role in osmoregulation and responding to stress tolerance (Xue et al. 2011; Xue et al. 2013; Joudi et al. 2012). Fructans are also considered as an important contributor to the total amount of fermentable oligosaccharides, disaccharides, monosaccharides and polyols (often termed FODMAPs). The term FODMAP was first coined by Gibson and Shepherd (2005) based on its relationship with Crohn's disease. FODMAPs are widely present in plants such as cereals as well as fruits and vegetables (Fraberger et al. 2018).

As wheat is considered a staple food crop and is consumed in large amounts globally (Van Loo et al. 1995; Moshfegh et al. 1999), it is considered as a major contributor of FODMAPs in our diet (Biesiekierski et al. 2011; Whelan et al. 2011). The type of FODMAPs present in wheat grain include monosaccharides (fructose), disaccharides (sucrose and maltose), trisaccharides (raffinose) fructo-oligosaccharides (fructans) as well as sugar alcohols (glycerol and mannitol) (Costabile et al. 2014; Gibson and Shepherd 2010). Fructans or fructo-oligosaccharides are the most abundant type of FODMAPs present in wheat grain. Wheat fructans are of graminan (branched)-type, with both $\beta-(2,1)$ and $\beta$ $(2,6)$ linkages, comprising mainly of fructose units with some having one glucose unit as well (Carpita et al. 1991). Of the different wheat grain fractions, the bran layer has the highest FODMAP content followed by "middlings" and then the endosperm (Knudsen 1997; Haskå et al. 2008). The concentration of fructans in whole wheat flour ranges from $0.66-3.20 \mathrm{~g} / 100 \mathrm{~g}$ and is a moderately heritable trait (Andersson et al. 2013; Huynh et al. 2008b).

FODMAPs have been associated with both positive and negative health effects. Fructan consumption has been shown to promote a healthy gut and improve immune function (Lomax and Calder 2008) as well as improve mineral bioavailability (Abrams et al. 2005; Abrams et al. 2007). Moreover, fructans have recently been recognized as dietary fibre or a prebiotic (Andersson et al. 2013). FODMAPs were also initially reported to be the cause of Crohn's disease and more recently, have been recognized as one of the major triggers of irritable bowel syndrome (Gibson and Shepherd 2010; Staudacher et al. 2011). This combination of positive and negative health effects of FODMAPs in human provides an opportunity to develop wheat varieties that cater to the needs of those who require high or low FODMAP diets.

A number of research studies have been published describing the type and quantity of FODMAPs found in various food sources as well as the role of FODMAPs in human health (Békés 2012b; Halmos et al. 2015; Haskå et al. 2008; Muir et al. 2014; Pirkola et al. 2018; Roberfroid et al. 2010; Struyf et al. 2018). There remains a lack of detailed information about the effect of genetic and environmental factors on fructan contents of wheat and wheat relatives. This has initiated large screening programs to identify geno- 
types with suitable (high or low) fructan contents to be utilised in breeding programs to produce varieties that will deliver specific health outcomes.

Identification of wheat varieties with specific characteristics such as low or high fructan contents will provide a platform for breeding wheat cultivars targeted to specific domestic and export markets seeking specific health outcomes. The aim of the current study was to screen a set of diverse Australian wheat varieties for their fructan contents to assess their importance in contributing to FODMAP levels in diets. This study also evaluates the importance of seasonal variation on fructan levels in Australian wheat varieties.

\section{Materials and Methods}

\section{Sample collection and preparation}

A set of 78 wheat varieties released in Australia between 1860 and 2015 was selected for the current study (Table S1*). All varieties were cultivated at the same location (Collingullie, New South Wales, Australia) over two growing seasons (2015 and 2016). Each variety was grown without irrigation with a plot size of $4.5 \mathrm{~m}^{2}$ with sowing and harvesting occurring in May and December, respectively. All field experiments were conducted by Australian Grain Technologies Limited (AGT).

Whole grain samples were ground to a particle size of approximately $0.5 \mathrm{~mm}$ using a laboratory mixer mill (Retsch MM 440). Total dry matter contents were determined using Mininfra SmarT NIR instruments. All samples were packed in paper bags, and used for analysis within 1 week.

\section{Fructan extraction and analysis}

Flour samples $(1 \mathrm{~g})$ were suspended in $40 \mathrm{~mL}$ distilled water $\left(80-85^{\circ} \mathrm{C}\right)$ and continuously mixed using a hotplate, magnetic stirrer (approximately $80^{\circ} \mathrm{C}$ ) for $15 \mathrm{~min}$ in order to completely solubilize fructans. The wheat/water mixture was cooled down to room temperature, adjusted to $50 \mathrm{ml}$ with distilled water and centrifuged at $480 \mathrm{~g}$ for $5 \mathrm{~min}$. Aliquot of supernatant was filtered through Whatman No. 1 filter paper and analysed immediately. The total fructan contents were determined according to the method described in Megazyme HK Assay Procedure (Mccleary et al. 2000; Megazyme fructan assay kit). All measurements were conducted in triplicate.

\section{Statistical analyses}

ANOVA for multiple comparisons (LSD test) was performed using SPSS (Version 25 IBM Corporation 1989, 2017.)

\footnotetext{
*Further details about the Electronic Supplementary Material (ESM) can be found at the end of the article.
} 


\section{Results}

A set of 78 Australian wheat varieties released in Australia between 1860 and 2015 were analysed for their fructan contents and results are presented in Fig. 1, Table S1.

Based on the statistical analysis, significant difference was observed between the overall mean fructan contents of the samples grown in the two consecutive seasons. The total fructan contents ranged from $1.01 \%-1.99 \%$ and $1.31 \%-2.27 \%$ in 2015 and 2016 , respectively. Analysis of variance carried out on the data indicated a small effect of genetic factors $(F=1.755, p=0.022)$, and very strong effects of harvest year $(F=97.855$, $\mathrm{p}=0.000)$ on fructan content.

Despite the significant differences in fructan contents over the two cultivation years, the grain fructan contents of 2015 and 2016 harvest were positively correlated (Fig. 2, $r=0.57, p \leq 0.01)$.

No significant genotype by environment interaction was observed in the current study, $(\mathrm{F}=0.475, p=0.998)$.

Furthermore, based on the average fructan levels of the two cultivation seasons, a number of varieties have been identified having higher or lower fructan levels. Varieties Suntop, Calingiri and Kite were found to have consistently lowest fructan levels (1.41, $1.41 \%$ and $1.53 \%$, respectively) while Batavia, Ega-gregory and Tincurrin $(4.21 \%, 3.78 \%$ and $3.67 \%$, respectively) seemed to consistently contain the highest levels of fructan.

Comparing the differences in the fructan levels in the corresponding samples from the two cultivation seasons, large variations were observed. In the case of the variety Chara practically no alteration can be observed (1.67\% and 1.68\% during 2015 and 2016 respectively), while a difference of $1.01 \%$ was found in the samples of the variety Excalibur grown in the two different seasons (1.22 and 2.23\% for 2015 and 2016, respectively).

\section{Discussion}

In recent years, the quantification of fructan in cereal grains is getting more attention due to extended health benefits and the adaptation of the dietary fibre definition. The amount of fructan in cereals is quite variable with highest amount on dry basis in rye (3.6-6.6\%) followed by wheat $(0.7-2.9 \%)$, spelt, durum, einkorn and triticale almost similar in their fructan contents (Verspreet et al. 2015). However, wheat is considered at the top due to its high consumption and therefore more contribution of total fructan content in diet compared to other cereals. In our study, the range of fructan contents observed in Australian wheat varieties are in line with the findings of (Andersson et al. 2013; Gélinas et al. 2016; Huynh et al. 2008; Verspreet et al. 2012; Ziegler et al. 2016) who reported fructan concentration from 0.7 to $2.9 \mathrm{~g} / 100 \mathrm{~g}$ of dry mass in bread and durum wheat.

The significant difference observed in grain fructan contents of the two growing seasons may be related to the moderate dry year observed in 2015 with lower than the average rainfall $(587.3 \mathrm{~mm})$ resulting in moisture stress condition. The 2016 growing season was cooler with a higher rainfall $(827.3 \mathrm{~mm})$ which resulted in higher fructan contents. The results reported here are in agreement with the findings of Huynh et al. (2008b) who 


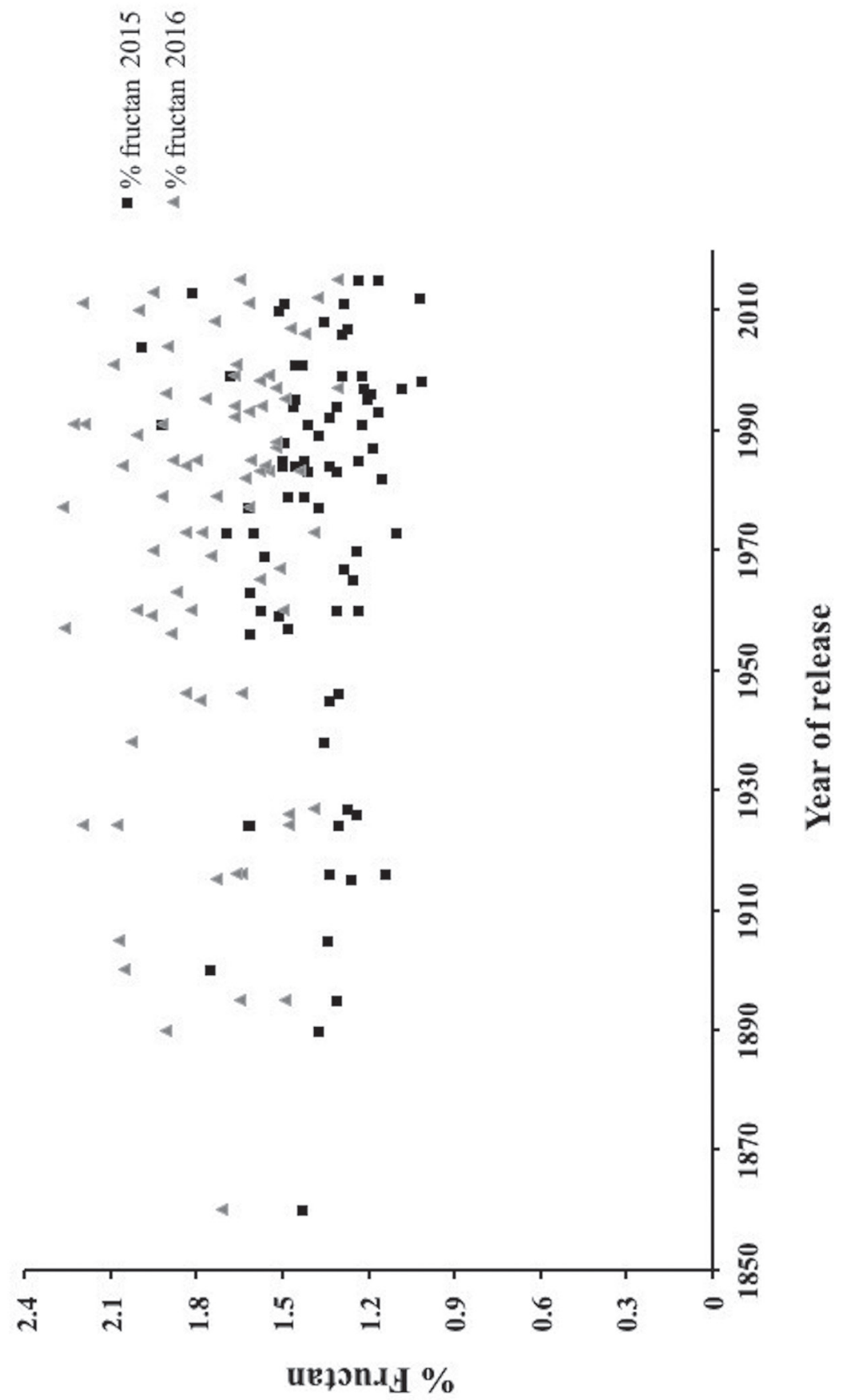

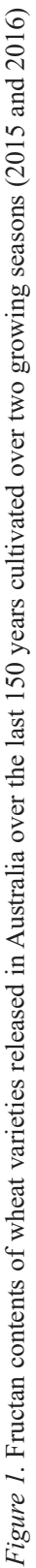




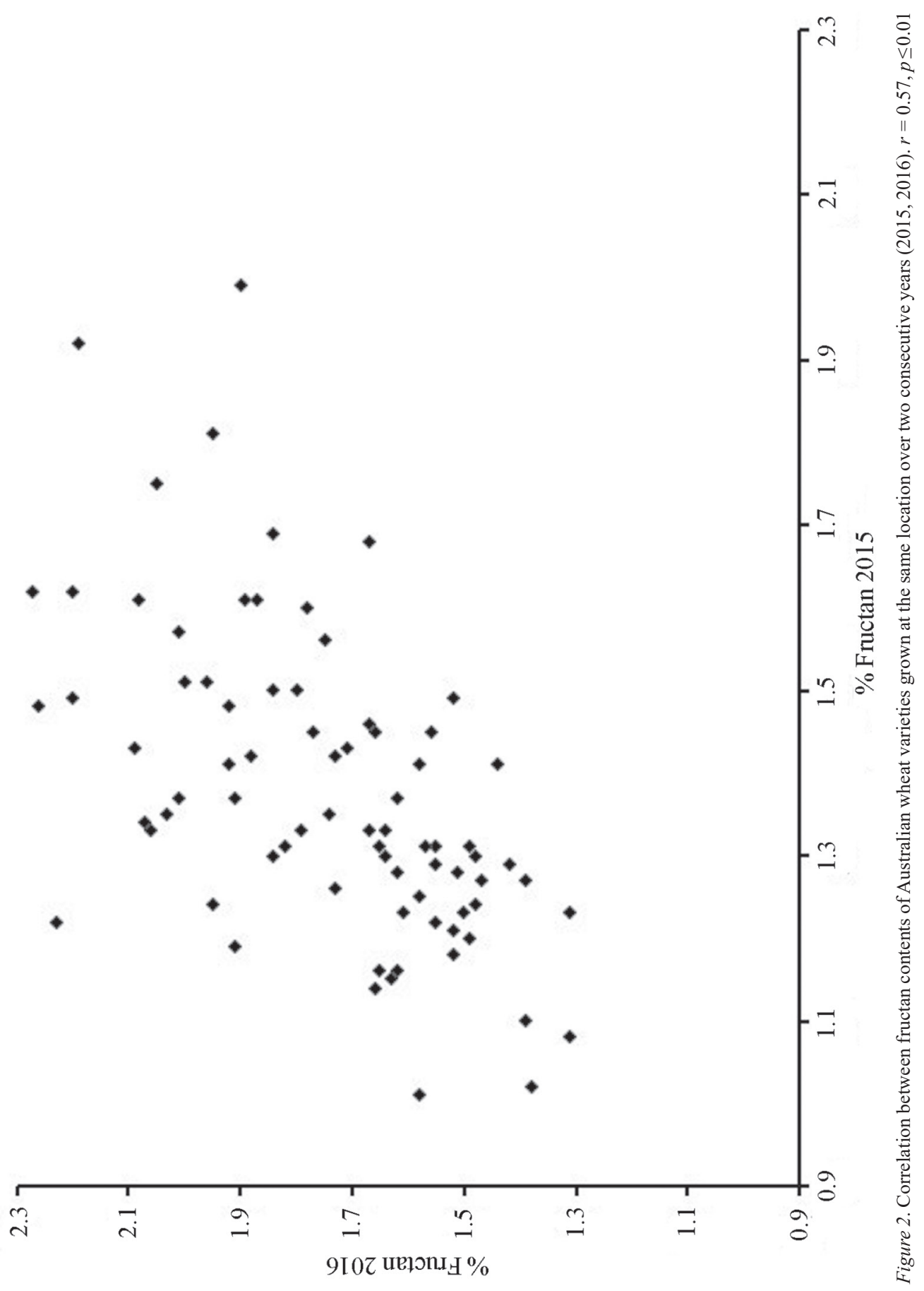


also observed significant changes in wheat grain fructan contents grown over two consecutive years $(2005,2006)$ and also suggested that moisture stress was a possible factor in determining fructan contents. However, Ziegler et al. (2016), Brandolini et al. (2011) who investigated the effect of environment and location on total fructan contents in wheat grain observed no significant changes. Huynh et al. (2008a) reported lower fructan contents in wheat grown in glasshouse $(0.7-1.6 \%)$ compared to the one grown under field conditions (1.5-2.3\%). In this study, it was suggested that low light intensity along with an ample supply of nutrients and water in the glasshouse which favoured the vegetative growth of the plants resulted in lower levels of grain fructans.

At the same time, the genotypic variation and moderate to high heritability of fructans in wheat opens perspectives for increasing kernel fructan concentration by gene regulation. Similar observations have been made in several other studies with some reporting the heritability of fructan contents in wheat as 0.64-0.94 (Huynh et al. 2008b; Huynh et al. 2008a; Ziegler et al. 2016). When selecting candidate lines for breeding programs to either increase or reduce fructan contents, not only the existence of high or low fructan content but also the consistency of these levels over different cultivation seasons should be considered. Wheat varieties with consistently very high and low fructan concentration have been identified in our study suggesting that these varieties could be bred for desirable fructan content.

Industry and consumers demands are driving a need to develop grain varieties which are not only more productive but also produce grains that can be utilized as a source material for healthy foods (Békés 2012a; Békés 2012b). As a consequence, the relative importance of compounds with health benefits such as fructans, has considerably increased in recent years (Shewry and Hey 2015). Hence, the importance of understanding the genetic and environmental factors that control the abundance of health-related compounds is increasing.

Based on the results presented here, breeding targets employed in Australia over the past 150 years have not appeared to have had an impact on fructan levels in the released varieties. Although varieties with relatively high (Batavia, Ega-gregory and Tincurrin) and low (Suntop, Calingiri and Kite) fructan contents have been identified. This suggests that selecting for higher yielding varieties with adequate bread-making quality does not favour higher or lower fructan levels. Based on this assumption, the possibility may exist to develop varieties with higher or lower fructan levels without compromising yield. This provides the opportunity to employ marker assisted breeding using the quantitative trait loci (QTLs) affecting grain fructan concentration in wheat identified by Huynh et al. (2008b) as a tool to develop premium wheat varieties with specific fructan levels.

\section{Acknowledgements}

This work was supported within project OTKA-K 16-119835, funded by the National Research, Development and Innovation Office, Hungary. 


\section{References}

Abrams, S.A., Griffin, I.J., Hawthorne, K.M., Liang, L., Gunn, S.K., Darlington, G., Ellis, K.J. (2005) ‘A combination of prebiotic short and long-chain inulin-type fructans enhances calcium absorption and bone mineralization in young adolescents', Am. J. Clin. Nutr. 82(2):471-476.

Abrams, S.A., Hawthorne, K.M., Aliu, O., Hicks, P.D., Chen, Z., Griffin, I.J. 2007. An inulin-type fructan enhances calcium absorption primarily via an effect on colonic absorption in humans. J. Nutr. 137(10):22082212.

Andersson, A.A., Andersson, R., Piironen, V., Lampi, A.-M., Nyström, L., Boros, D., Fraś, A., Gebruers, K., Courtin, C.M., Delcour, J.A. 2013. Contents of dietary fibre components and their relation to associated bioactive components in whole grain wheat samples from the HEALTHGRAIN diversity screen. Food Chem. 136(3-4):1243-1248.

Békés, F. 2012a. New aspects in quality related wheat research: 1. Challenges and achievements. Cereal Res. Commun. 40(2):159-184.

Békés, F. 2012b. New aspects in quality related wheat research: II. New methodologies for better quality wheat. Cereal Res. Commun. 40(3):307-333.

Belderok, B. 2000. The wheat grain. In: Mesdag, J. and Donner, D.A. (eds), Bread-making quality of wheat: a century of breeding in Europe. Springer Science \& Business Media, pp. 15-18.

Biesiekierski, J., Rosella, O., Rose, R., Liels, K., Barrett, J., Shepherd, S., Gibson, P., Muir, J. 2011. Quantification of fructans, galacto-oligosacharides and other short-chain carbohydrates in processed grains and cereals. J. Hum. Nutr. Diet. 24(2):154-176.

Brandolini, A., Hidalgo, A., Plizzari, L., Erba, D. 2011. Impact of genetic and environmental factors on einkorn wheat (Triticum monococcum L. subsp. monococcum) polysaccharides. J. Cereal Sci. 53(1):65-72.

Carpita, N.C., Housley, T.L., Hendrix, J.E. 1991. New features of plant-fructan structure revealed by methylation analysis and carbon-13 nmr spectroscopy. Carbohyd. Res. 217:127-136.

Costabile, A., Santarelli, S., Claus, S.P., Sanderson, J., Hudspith, B.N., Brostoff, J., Ward, J.L., Lovegrove, A., Shewry, P.R., Jones, H.E. 2014. Effect of breadmaking process on in vitro gut microbiota parameters in irritable bowel syndrome. PLoS One 9(10):e111225.

Fraberger, V., Call, L.-M., Domig, K., D’Amico, S. 2018. Applicability of yeast fermentation to reduce fructans and other FODMAPs. Nutrients 10(9):1247.

Gélinas, P., McKinnon, C., Gagnon, F. 2016. Fructans, water-soluble fibre and fermentable sugars in bread and pasta made with ancient and modern wheat. Int. J. Food Sci. Tech. 51(3):555-564.

Gibson, P., Shepherd, S. 2005. Personal view: food for thought-western lifestyle and susceptibility to Crohn's disease. The FODMAP hypothesis. Aliment. Pharm. Ther. 21(12):1399-1409.

Gibson, P.R., Shepherd, S.J. 2010. Evidence-based dietary management of functional gastrointestinal symptoms: the FODMAP approach. J. Gastroen. Hepatol. 25(2):252-258.

Halmos, E.P., Christophersen, C.T., Bird, A.R., Shepherd, S.J., Gibson, P.R., Muir, J.G. 2015. Diets that differ in their FODMAP content alter the colonic luminal microenvironment. Gut 64(1):93-100.

Haskå, L., Nyman, M., Andersson, R. 2008. Distribution and characterisation of fructan in wheat milling fractions. J. Cereal Sci. 48(3):768-774.

Huynh, B.-L., Palmer, L., Mather, D.E., Wallwork, H., Graham, R.D., Welch, R.M., Stangoulis, J.C. 2008 a. Genotypic variation in wheat grain fructan content revealed by a simplified HPLC method. J. Cereal Sci. 48(2):369-378.

Huynh, B.-L., Wallwork, H., Stangoulis, J.C., Graham, R.D., Willsmore, K.L., Olson, S., Mather, D.E. 2008 b. Quantitative trait loci for grain fructan concentration in wheat (Triticum aestivum L.). Theor. Appl. Genet. 117(5):701-709.

Joudi, M., Ahmadi, A., Mohamadi, V., Abbasi, A., Vergauwen, R., Mohammadi, H., Van den Ende, W. 2012. Comparison of fructan dynamics in two wheat cultivars with different capacities of accumulation and remobilization under drought stress. Physiol. Plantarum 144(1):1-12.

Knudsen, K.E.B. 1997. Carbohydrate and lignin contents of plant materials used in animal feeding. Anim. Feed Sci. Tech. 67(4):319-338. 
Lomax, A.R., Calder, P.C. 2008. Prebiotics, immune function, infection and inflammation: a review of the evidence. Brit. J. Nutr. 101(5):633-658.

Mccleary, B.V., Murphy, A., Mugford, D.C. 2000. Measurement of total fructan in foods by enzymatic/spectrophotometric method: collaborative study. J. AOAC Int. 83(2):356-364.

Megazyme fructan assay kit. Megazyme, Ireland: Megazyme. Available at: https://secure.megazyme.com/files/ Booklet/K-FRUC_DATA.pdf (Accessed: 19-11-2018).

Moshfegh, A.J., Friday, J.E., Goldman, J.P., Ahuja, J.K.C. 1999. Presence of inulin and oligofructose in the diets of Americans. The Journal of nutrition 129(7):1407S-1411S.

Muir, J., Mills, J., Suter, D., Bekes, F., Liels, K., Yao, C., Gibson, P. 2014. Reduced FODMAPs in gluten-free grains may explain the improved symptoms in people with IBS following a gluten-free diet. Journal of Nutrition \& Intermediary Metabolism 1:14-15.

Pirkola, L., Laatikainen, R., Loponen, J., Hongisto, S.-M., Hillilä, M., Nuora, A., Yang, B., Linderborg, K.M., Freese, R. 2018. Low-FODMAP vs regular rye bread in irritable bowel syndrome: Randomized SmartPill@ study. World J. Gastroentero. 24(11):1259.

Roberfroid, M., Gibson, G.R., Hoyles, L., McCartney, A.L., Rastall, R., Rowland, I., Wolvers, D., Watzl, B., Szajewska, H., Stahl, B. 2010. Prebiotic effects: metabolic and health benefits. Brit. J. Nutr. 104:S1-S63.

Shewry, P.R., Hawkesford, M.J., Piironen, V., Lampi, A.-M., Gebruers, K., Boros, D., Andersson, A.A., Åman, P., Rakszegi, M., Bedo, Z. 2013. Natural variation in grain composition of wheat and related cereals. J. Agr. Food Chem. 61(35):8295-8303.

Shewry, P.R., Hey, S.J. 2015. The contribution of wheat to human diet and health. Food and Energy Security 4(3): 178-202.

Staudacher, H.M., Whelan, K., Irving, P.M., Lomer, M.C. 2011. Comparison of symptom response following advice for a diet low in fermentable carbohydrates (FODMAPs) versus standard dietary advice in patients with irritable bowel syndrome. J. Hum. Nutr. Diet. 24(5):487-495.

Struyf, N., Verspreet, J., Courtin, C.M. 2018. FODMAP reduction in yeast-leavened whole wheat bread. Cereal Food. World 63(4):152-154.

Van den Ende, W., El-Esawe, S.K. 2014. Sucrose signaling pathways leading to fructan and anthocyanin accumulation: a dual function in abiotic and biotic stress responses? Environ. Exp. Bot. 108:4-13.

Van Loo, J., Coussement, P., De Leenheer, L., Hoebregs, H., Smits, G. 1995. On the presence of inulin and oligofructose as natural ingredients in the western diet. Crit. Rev. Food Sci. 35(6):525-552.

Verspreet, J., Pollet, A., Cuyvers, S., Vergauwen, R., Van den Ende, W., Delcour, J.A., Courtin, C.M. 2012. A simple and accurate method for determining wheat grain fructan content and average degree of polymerization. J. Agr. Food Chem. 60(9):2102-2107.

Verspreet, J., Dornez, E., Van den Ende, W., Delcour, J.A., Courtin, C.M. 2015. Cereal grain fructans: structure, variability and potential health effects. Trend Food Sci. Tech. 43(1):32-42.

Whelan, K., Abrahmsohn, O., David, G.J., Staudacher, H., Irving, P., Lomer, M.C., Ellis, P.R. 2011. Fructan content of commonly consumed wheat, rye and gluten-free breads. Int. J. Food Sci. Nutr. 62(5):498-503.

Xue, G.-P., Drenth, J., Glassop, D., Kooiker, M., McIntyre, C.L. 2013. Dissecting the molecular basis of the contribution of source strength to high fructan accumulation in wheat. Plant Mol. Biol. 81(1-2):71-92.

Xue, G.P., Kooiker, M., Drenth, J., McIntyre, C.L. 2011. TaMYB13 is a transcriptional activator of fructosyltransferase genes involved in $\beta$-2, 6-linked fructan synthesis in wheat. Plant J. 68(5):857-870.

Ziegler, J.U., Steiner, D., Longin, C.F.H., Würschum, T., Schweiggert, R.M., Carle, R. 2016. Wheat and the irritable bowel syndrome-FODMAP levels of modern and ancient species and their retention during bread making. J. Funct. Food 25:257-266.

\section{Electronic Supplementary Material (ESM)}

Electronic Supplementary Material (ESM) associated with this article can be found at the website of CRC at http://www.akademiai.com/content/120427/

Electronic Supplementary Table S1. Fructan contents in whole grain flours of wheat varieties released in Australia between 1860-2015

Cereal Research Communications 47, 2019 